\title{
Comments on 'On the equivalence of causal LTI iterative learning control and feedback control'
}

The area of iterative learning control (ILC) now has a large and increasing body of research with an increasing number of applications (supported by a sizable number of actual experimental verification studies). The paper (Goldsmith, 2002) contains a number of assumptions and technical errors that invalidate its conclusion that normal feedback control is preferable to causal ILC, and the purpose of this note is to state the problems with this paper and clearly articulate the technical and practical validity of the ILC concept.

Of course, causal ILC (in the sense of Goldsmith (2002)) is not the only option for the community and, implicitly, non-causal control structures may improve performance and other desirable closed-loop properties. Indeed, see, for example, Amann, Owens, and Rogers (1998), there is already much published work which (as one possible choice) uses optimal control concepts to produce convergent ILC algorithms that use current trial feedback plus previous trial (non-causal in the classical sense) feedforward actions. The central issue that we wish to challenge is that the proposed equivalence between causal ILC and feedback control invalidates the former and in what follows we give the details for the 4 main areas we challenge.

\section{The use of $[0, T]$ versus $[0, \infty]$ - two very different cases}

The analysis in Goldsmith (2002) is in $L_{2}(0, \infty)$ rather than the ILC situation of $L_{2}(0, T)$ where $T$ is finite. At a simple theoretical level, the second choice is more realistic as it reflects the fact that the iterations are undertaken on a finite interval $[0, T]$ and it does not suffer from the technical difficulty (in $L_{2}(0, \infty)$ ) that it is necessary that all signals tend to zero as time $t$ tends to infinity. At a deeper level, there are very serious technical issues that separate these two situations as articulated in the literature on repetitive processes, such as Rogers and Owens (1992) (from which the following analysis is extracted).

The theory required is based on the analysis of recursive structures of the form

$y_{k+1}=L_{T} y_{k}+d_{T}$, with signals $y_{k}$ in some Banach space (say, $S_{T}=L_{p}^{m}(0, T)$ ) and $L_{T}$ a bounded linear operator from $S_{T}$ into itself. The parameter $T$ is called the pass length and normally represents a time or spatial interval of interest and is finite and fixed. The key results are follows: for a fixed value of $T$, the recursive system is asymptotically stable if, and only if, the recursive equation has a limit for any choice of initial point $y_{0}$ and sufficiently small perturbations to the operator $L_{T}$. It is in effect a form of robust stability that guarantees that the stable set is open (in the operator norm topology). The condition for asymptotic stability is that $r\left(L_{T}\right)<1$, where $r(\cdot)$ is the spectral radius of the argument. Under these conditions, the recursion converges with a limit

$y_{\infty}=\left(I-L_{T}\right)^{-1} d_{T}$.

A concept of stability that applies to the case where $T \rightarrow$ $\infty$ is called "stability along the pass" and can be stated as the requirement that, for some $\lambda<1$

$$
\sup _{\eta \mid>\lambda, T>0}\left\|\left(\eta I-L_{T}\right)^{-1}\right\|<\infty .
$$

In essence, it is required that the limit be uniformly bounded over $T$ even in the presence of perturbations of $L_{T}$ (Rogers \& Owens, 1992). A simple summary of the issue is that the stability condition for a repetitive or iterative system is not continuous as $T \rightarrow \infty$. As a consequence, conclusions based upon an $[0, \infty]$ analysis generally do not apply to the finite interval case! Consider now the simple iterative system in $\mathbb{R}^{n}$ :

$\dot{x}_{k+1}(t)=A x_{k+1}(t)+B_{0} y_{k}(t)+d$,

$y_{k+1}(t)=C x_{k+1}+D y_{k}(t)$,

$x_{k+1}(0)=x(0)$.

Using the concepts of asymptotic stability reported in Rogers and Owens (1992), the iterative system is asymptotically stable when $T$ is finite if, and only if, $r(D)<1$. In contrast the iterative system is stable when $T$ is infinite if, and only if,

$\sup _{\omega \geqslant 0} r\left(G_{0}(i \omega)\right)<1, G_{0}(s)=C\left(s I_{n}-A\right)^{-1} B_{0}+D$.

For single-input, single-output (SISO) systems, this condition reduces to the requirement that $\left\|G_{0}\right\|<1$, where $\|\cdot\|$ 
denotes the $H_{\infty}$ norm, which relates to, but predates, the work in Goldsmith (2002). In particular, a central part of the claimed new insight in Goldsmith (2002) has been known for a decade (Rogers \& Owens, 1992) and more (Padieu \& $\mathrm{Su}, 1990$ ) (see also below where other results/ideas from Padieu and $\mathrm{Su}$ (1990) are also employed).

As $D=G_{0}(\infty)$, stability on finite intervals is a necessary but not sufficient condition for stability on infinite intervals. The very different nature of the stability condition for finite and infinite intervals is very clear in both mathematical and dynamics terms. In particular, the stability conditions underline the insensitivity of stability on finite intervals to the details of systems dynamics and clearly state the dependence of stability on an infinite interval on the details of plants dynamics. In both cases, the iterative system converges to a limit so both satisfy the main requirement for application to ILC. The absolutely critical issue is that the most appropriate case is used - in Goldsmith (2002) it is the inappropriate one that is used.

One major difference arising from the two cases needs detailed analysis and is illustrated by the following example - $\operatorname{set} A=-1, B_{0}=1+\beta, C=1$ and $D=0$ in (4) and note the iterative system is stable on any finite time $T$. Also sequence $\left\{y_{k}\right\}$ converges in $L_{2}(0, T)$ to a limit $y_{\infty}$ satisfying

$\dot{y}_{\infty}(t)=\beta y_{\infty}(t)+1, \quad y_{k+1}=0$.

This limit exists on all finite intervals and, in that sense, exists on $[0, \infty]$. It is, however, always bounded in $L_{2}(0, T)$ but potentially unbounded on $L_{2}(0, \infty)$ as its classical stability depends on $\beta$. More precisely, (6) is stable if, and only if, $\beta<0$ which is precisely the condition for the $H_{\infty}$ norm of $G_{0}(s)$ to be less than one.

The outcome of the above discussion is that great care must be taken when discussing ILC to clearly identify and justify the use of finite or infinite interval analysis. The two cases are entirely different in terms of stability and there are very clear differences in the interpretation of the limits of an iterative or repetitive process - the analysis in Goldsmith (2002) ignores this completely.

To demonstrate some of the major problems that can arise in ILC analysis, consider Goldsmith (2002, Section 2) which proceeds as follows:

(1) Characterizes the process by the equation $\mathrm{y}=\mathrm{Pu}$ in $L_{2}(0, \infty)$ which implicitly assumes and requires zero initial conditions and a stable-process model. In contrast, analysis in $L_{2}(0, T)$ does not require open loop stability for functional analytic consistency.

(2) Characterizes the limit of his iteration by $u_{d} \in L_{2}(0, \infty)$. This is a serious limitation in his analysis as the symbolic solution of the problem $u_{d}=P^{-1} y_{d}$ indicates that it is generically necessary that the plant is minimum phase for $u_{d} \in L_{2}(0, \infty)$ to be true. His analysis therefore excludes the case of non-minimum phase systems.
Similar comments hold true for the assumption in Goldsmith (2002) that $u_{\infty} \in L_{2}(0, \infty)$.

The effect of these observations is, at very best, to severely constrain the analysis of Goldsmith (2002) and completely invalidate the interpretation placed on the results obtained.

\section{Equivalent feedback control-is the idea meaningful?}

It is trivially true that any convergent iterative process described by a (continuous) recursion

$u_{k+1}=g\left(u_{k}, e_{k+1}, e_{k}\right), \quad u_{0} \quad$ specified

converges to a limit satisfying

$u_{\infty}=g\left(u_{\infty}, e_{\infty}, e_{\infty}\right)$.

If this implicit equation can be solved for a causal (in whatever sense) solution, $u_{\infty}=f\left(e_{\infty}\right)$ a causal feedback is potentially created that, in principle, can be applied directly. This control input will generate the limit dynamics $e_{\infty}$ directly without iteration. The observation that there may exist a causal equivalent feedback control that generates the final error immediately is hence essentially trivial and, in effect, has been known for a long time in repetitive process theory - the step from there to ILC (which, in turn, motivates the whole approach of Goldsmith (2002)) is therefore nothing new. The real issues are (i) the convergence of the process and any constraints that this might place on ILC algorithm and/or plant dynamics, (ii) the nature and acceptability of the limit dynamics, and (iii) if feedback control is to be considered as an alternative, the practical acceptability of the equivalent feedback algorithm. Situations also exist where the map $f$ does not exist (e.g. when $F=I$ in Goldsmith (2002) and, consequently, an equivalent control does not exist.

\section{The "Lemma 2" stability condition in Goldsmith (2002)}

We note first that the concept of internal stability presented by Goldsmith (2002) has little meaning, particularly when $T$ is finite as boundedness in finite time is always guaranteed trivially. In infinite time, the assumption of the existence of an input $u_{d} \in L_{2}(0, \infty)$ also excludes application to non-minimum-phase systems as discussed above.

In addition, Definition 2 and Lemma 2 of Goldsmith (2002) clearly demonstrate the limitations of this work. The conclusion that a sufficient condition for convergence is that $\mathrm{PH}$ is stable and $\|H\|<1$ is indeed valid but the following points are relevant.

(a) The convergence condition $\|H\|<1$ is exactly the requirement that the $H_{\infty}$ norm of the transfer function matrix of $H$ is less than unity. On finite time $(T<\infty)$ results 
from Rogers and Owens (1992) can be used leading to the conclusion that this condition is replaced by the requirement that the "D" matrix in a proper realization of $H$ should have spectral radius strictly less than unity. This is a simpler condition with the added advantage that the spectral radius condition is both necessary and sufficient if stability is to be retained under sufficiently small perturbations of the plant model.

(b) More seriously, Lemma 2 in Goldsmith (2002) claims a necessary ("only if") convergence condition based on a requirement that $M(s)=I-H(s)$ be positive real. Although there may be situations where this result (or some similar result (see below)) is correct for SISO systems on an infinite time interval $T=\infty$, the case for a finite interval $T<\infty$ is more complex. Again, using the results from Rogers and Owens (1992), the recursion $v_{i+1}=H v_{i}$ is asymptotically stable in $L_{2}(0, T)$ if, and only if, the scalar "D matrix" satisfies $|D|<1$. As there are examples where this condition is satisfied but the condition $\|H\|<1$ in Goldsmith (2002) is not satisfied, it follows that the Lemma stated in this paper is incorrect. A correct alternative version is given in Lemma 1 below. The necessary and sufficient condition for (robust) stability stated above can be extended (in part) to the situation where $D=1$ (see also Padieu and Su (1990)) where $[0, \infty]$ is considered to give sufficient conditions for convergence as $\|H\| \leqslant 1$ and all frequencies where $|H(i \omega)|=1$ are isolated. The ideas are clearly related to the claimed results in Goldsmith (2002) but they are not the sameagain we see that this work ignores previous and correct contributions to the field. Also if $D=1$, then $H^{-1}=I+$ $M_{0}$ where $M_{0}$ is strictly proper and hence the following result.

Lemma 1. A sufficient condition for ILC convergence in $L_{2}(0, \infty)$ is that $\|H\| \leqslant 1$ which is achieved (a sufficient condition) if $M_{0}=H^{-1}-I \in \Omega$ (i.e. $M_{0}$ is positive real).

This result has a structural similarity to that of Goldsmith (2002), but differs in the use of the inverse and the replacement of a necessary condition from the work under discussion here by a sufficient condition (derived from Padieu and $\mathrm{Su}(1990)$ ). It is our belief that this is the true result based on a combination of a norm condition with a positive real condition.

(c) The condition that $M_{0}$ is strictly proper and positive real requires it to have a relative degree of unity and to be minimum phase and stable is not true for a strictly proper plant $P$, where $C$ and $D$ are proper and $P$ has relative degree 2 or more and/or it is non-minimum phase. Any consequent conclusions based on this result therefore only apply to plants from this (restrictive) class.

The conclusion reached from the above discussion is that the results in Goldsmith (2002) are incorrect and that better results have been available in the literature for some time.

\section{The nature of the limit and the validity of the feedback equivalence claims}

The existence of a limit error is most certainly necessary for acceptability of an ILC algorithm but an absolutely essential requirement is that this limit error $e_{\infty}$ has an acceptable form. The paper (Goldsmith, 2002) is correct in stating that (Lemma 3$) e_{\infty}=0$ if $F=I$, but fails to note that the existence of a limit error $e_{\infty}$ does not imply that this error will be acceptable - see the simple example given above with $\beta>0$. A more compelling example can be found in an ILC context. If $D=0$ and $F$ is a scalar, then

$e_{k+1}=\left(I+(P C)^{-1}\right)\left(F e_{k}+(1-F) y_{d}\right)$.

If $P C$ is strictly proper, then the relevant "D-matrix" is just $F I$ and hence a limit error exists on any $L_{2}(0, T)$ (with $T$ finite but arbitrary) if, and only if, $|F|<1$ and also

$e_{\infty}=\left(I+P C_{\text {eff }}\right)^{-1} y_{d}, C_{\text {eff }}=\frac{C}{1-F}$,

i.e. the limit error is generated from an equivalent unity negative feedback control $C_{\text {eff }}$. Hence a necessary condition for the limit error to be small is that $C_{\text {eff }}$ is very high gain! Root-locus analysis also shows that this condition is not sufficient. In particular, if $P C_{\text {eff }}$ has relative degree greater than or equal to two and/or it is non-minimum phase, $e_{\infty}$ is a potentially unstable and/or highly oscillatory signal. Again the existence of a limit and its acceptability are unrelated and are only related in a satisfactory way if the plant satisfies some structural requirements - again these correct results/interpretations are consistent with long known repetitive process stability theory (Rogers \& Owens, 1992).

Turning again now to Goldsmith (2002), Section 2 of this paper centers on the main result stated in Theorem 2. The problems with this result are twofold

(a) It is based on the validity of Lemma 2 as a necessary condition for the existence of a limit which has been shown here to be incorrect.

(b) The result is based on the assumption that for each $v_{0} \in L, \lim _{k \rightarrow \infty} e_{k}=0$. This requires that $F=I$ (which presents no practical difficulties) but there are no known necessary and sufficient conditions for ILC stability in this case! Hence this main result is based on an assumption that cannot be checked in general. It can be checked in special cases, e.g. in the special case where $D=0$ (for simplicity), when $H=(I+P C)^{-1}$ which satisfies (Padieu \& Su, 1990) condition that $\|H\| \leqslant 1$ if $P C$ is positive real (a sufficient condition). This also shows (by a simple calculation) that the feedback $K=C+k C$ will lead to the desired high gain equivalence, $\lim _{k \rightarrow \infty} e_{k}=0$. Some reformulations of this result in Goldsmith (2002) therefore can be proved but, as in the above example, there will be constraints placed on the form and structure of the plant. The simplest form of constraint is that of being positive real excluding many situations of practical interest. 


\section{References}

Amann, N., Owens, D. H., \& Rogers, E. (1998). Predictive optimal iterative learning control. International Journal of Control, 69(2), 203-226.

Goldsmith, P. L. (2002). On the equivalence of causal LTI iterative learning control and feedback control. Automatica, 38, 703-708.

Padieu, F., \& Su, R. (1990). An $H_{\infty}$ approach to learning control systems. International Journal of Adaptive Control and Signal Processing, 4, 465-474.

Rogers, E., \& Owens, D. H. (1992). Stability analysis for linear repetitive processes. Lecture Notes in Control and Information Sciences Series, Vol. 175. Berlin: Springer.
D.H. Owens

Department of Automatic Control and Systems Engineering, University of sheffield, $U K$

E-mail address: d.h.owens@sheffield.ac.uk

\author{
E. Rogers
}

School of Electronics and Computer Science, University of Southampton, High field, Southampton S0171BJ, UK E-mail address: etar@ecs.soton.ac.uk. 